\title{
Le statut du paranormal dans Bouvard et Pécuchet
}

\author{
Chenoa Marshall \\ Université d'Ottawa
}

Pourquoi Gustave Flaubert a-t-il entrepris d'écrire son dernier roman, Bouvard et Pécuchet? Le projet a duré huit ans, lui a fait lire plus de 1500 volumes (voir Kempf, 1990, p. 39), et l'écrivain n'a cessé de se plaindre de l'énormité de la tâche. De plus, Flaubert était tourmenté par divers problèmes de santé qui l'empêchaient par moments d'écrire. Il avait également de graves problèmes financiers. Or, bien qu'il ait douté dès le départ de la réception de sa "farce», il continuait d'y travailler. La deuxième question que je vais aborder dans le cadre de cet article porte sur un segment d'une vingtaine de pages au milieu de cette compilation des savoirs écrits, segment qui traite du 
magnétisme, des tables tournantes, du swedenborgisme et de la magie. Quelle relation Flaubert entretenait-il avec le paranormal? S'il est facile de relever dans sa correspondance des exemples de scepticisme envers le paranormal, je tenterai de montrer que le roman se fait à ce sujet plus ambigu.

\section{Le réalisme et le paranormal}

Le projet de Bouvard et Pécuchet, conçu vers 1843 (Kempf, 1990, p. 31), n'est pas commencé avant 1872, quand Flaubert mène ses premières recherches bibliographiques. À plusieurs reprises, il est sur le point de renoncer au projet et, en mai 1875, il l'abandonne, pour ne le reprendre qu'en juin 1877. L'intervalle de trente ans entre la conception du projet et le début de l'entreprise soutient la thèse selon laquelle il s'agit d'un projet motivé principalement par une envie ancienne, qui a grandi avec les années. Dans les toutes premières esquisses de Bouvard et Pécuchet, les deux copistes devaient uniquement étudier des sujets agricoles (voir Flaubert, 1979, p. 8), mais, entre la conception et la rédaction, le projet prend de l'ampleur. Flaubert ajoute le paranormal, sans y attribuer une importance particulière, à la liste des sujets abordés par les deux protagonistes, en même temps que la philosophie, la médecine, la littérature et une multitude d'autres sujets, créant ainsi un roman quasi encyclopédique. Le seul fait d'y inclure le paranormal, un sujet devenu marginal après son apogée survenue trente ans auparavant, indique que Flaubert y attachait une certaine importance, surtout si l'on tient compte de l'aspect personnel du projet. 
Conformément aux exigences de la forme encyclopédique, Flaubert entreprend de faire de Bouvard et Pécuchet une œuvre aussi réaliste que possible. Ce réalisme s'exprime dans la création du texte à deux niveaux : le lieu et le contenu. Flaubert n'inclut aucune information qui n'existe pas dans un livre ou un autre document publié. Chaque parcelle d'information qui paraît à travers le roman a été puisée parmi les 1500 livres, en majorité de genre non littéraire (voir Charles, 2003, p. 18), consultés par l'auteur. En outre, chaque lieu qui paraît dans le roman trouve son contrepoint dans la réalité, dûment visitée par l'auteur. Flaubert «se sent[ait] incapable d'écrire sa première phrase sans avoir vérifié en nature "un certain paysage" qu'il a[vait] en tête » (Kempf, 1990, p. 109), et il parcourt ainsi le pays normand jusqu'à ce qu'il trouve la maison qu'il a imaginée. Rien dans cette création littéraire n'est laissé au hasard. Cependant, malgré cette obsession d'une mise en scène réaliste, Flaubert ne pratique pas un art qui imite la nature, mais crée un art qui copie l'art qui imite la nature. Meyer affirme que Flaubert «selects what to use, where to put it, and thereby comments on what he copies» (p. 41). Pourquoi inclure le paranormal et non les mathématiques (une science importante avec une histoire riche qui ne figure pas dans l'œuvre), sinon parce que Flaubert a ressenti le besoin particulier de se prononcer sur l'occulte? Quelle importance Flaubert attribue-t-il donc au paranormal?

\section{Le vocabulaire du savoir}

Un aspect qui renforce la vraisemblance ou la crédibilité du magnétisme et de la magie dans le segment paranormal est 
l'emploi d'un vocabulaire «scientifique». Le magnétisme déborde de termes techniques : " grands ou [...] petits courants, ascendantes ou descendantes, longitudinales, transversales » (Flaubert, 1979, p. 281). Ces termes correspondent à des techniques censées soigner les douleurs intercostales, la névrose de l'estomac, l'encéphaloïde, la phtisie ou l'hémiplégie. Intimidant dans sa technicité, ce vocabulaire donne un aspect scientifique au magnétisme en lui attribuant une vraisemblance et une autorité artificielles (voir Meyer, 1994, p. 43). La représentation relativement convaincante du magnétisme, un sujet que Flaubert inclut dans son catalogue personnel des imbécillités du XIX siècle au côté des escargots magiques et des choux géants (voir Wall, 2006, p. 145), remet en question toutes les formes du savoir scientifique, dont la validation repose, en dernière analyse, sur des effets de langage.

Avec la magie, la puissance du vocabulaire va encore plus loin. La maîtrise des mots y est égale au pouvoir : il faut posséder les mots "magiques» avant de pouvoir posséder le monde, car ceux-ci dénotent un écart sociétal entre la personne éduquée et l'ignorant, ou entre Flaubert et les bourgeois normands. La maîtrise apparente du magnétisme par Bouvard et Pécuchet, de même que la fluidité et la cohérence du vocabulaire savant qu'ils emploient, est particulière, si on la compare avec d'autres domaines du savoir qu'ils explorent. Par exemple, les deux personnages manifestent souvent une ignorance du lexique pertinent, notamment dans leur étude de l'anatomie. Après des journées d'étude, " ils démontaient complètement le cadavre, puis se trouvaient embarrassés pour remettre en place les morceaux» (p.121). Les organes humains, aussi mystérieux à Bouvard et Pécuchet qu'avant le début de leur étude anatomique, ne demeurent que des 
« morceaux » embarrassants, soulignant leur ignorance et leur incapacité de s'intéresser au sujet. Ailleurs, l'emploi d'un vocabulaire savant a pour effet final de semer la confusion. Par exemple, dans l'étude de l'arboriculture, Flaubert utilise des termes techniques non pas pour valider cette science fondée sur des traditions millénaires, mais bien pour semer la confusion. Pécuchet, face à la taille des arbres, « s'embrouilla dans les sur-mères, les sous-mères, et les deuxièmes sousmères. Des vides et des pleins se présentaient toujours où il n'en fallait pas. » (p. 96) Pécuchet est incapable d'appliquer ce qu'il a appris dans des livres d'arboriculture et l'utilisation de termes scientifiques hors du contexte original complique le sujet au lieu de l'éclaircir (Meyer, 1994, p. 39). Par contre, en matière de magnétisme, Bouvard et Pécuchet en maîtrisent le vocabulaire, interprétant et employant les différentes passes magnétiques, «biditiges, triditiges ou même quinditiges » (p. 281), ou «l'addigitation nasale » (p. 282), de manière à se convaincre eux-mêmes et à convaincre leur entourage : « Leurs manières onctueuses captèrent le monde » (p. 281). Même si leurs succès en magnétisme peuvent être attribués à des coïncidences, ils réussissent à saisir l'essentiel de cette pratique : la théâtralité et le charisme. La maîtrise par Bouvard et Péchuchet du lexique «scientifique» propre au magnétisme a pour effet de rendre le paranormal plus crédible, semble-t-il, que plusieurs des autres savoirs.

\section{Le paranormal dans la hiérarchie des savoirs}

Mais comment le paranormal est-il classé relativement aux autres savoirs? Sur le plan quantitatif, le segment paranormal 
est comparable aux autres. Le fait qu'il n'apparaît qu'au huitième chapitre, ou qu'il est précédé par une section sur la gymnastique, ne revêt aucune importance particulière. Aucune hiérarchie n'est impliquée dans l'ordre de la présentation, celuici étant fondé sur la facilité à créer une transition fluide entre les sujets. Les deux copistes abordent un sujet, s'informent au moyen d'une multitude de livres à la fois pertinents et contradictoires, essayent d'appliquer leur savoir nouvellement acquis et, désillusionnés par les incohérences ou distraits par leur curiosité pour un nouveau sujet, ils finissent par l'abandonner pour aborder le sujet suivant. Cette structure cyclique et répétitive a pour effet de mettre tous les savoirs sur un même plan.

Cela dit, toutes les manifestations paranormales à l'intérieur du segment en question ne sont pas traitées sur un pied d'égalité. Le magnétisme à lui seul occupe dix des dix-neuf pages, la magie quatre pages, les tables tournantes trois pages, et le swedenborgisme et la trompe aromale n'occupent qu'une petite page et demie. Ces deux derniers domaines occupent donc la place d'honneur sur l'échelle du scepticisme flaubertien. Le narrateur définit le swedenborgisme en disant qu'il "pose en dogme l'amélioration fatale de notre espèce » (p. 290). Selon Bouvard, ce n'est que le "délire d'un imbécile» (p. 292) et même Pécuchet «s'abîmait l'intellect pour comprendre ce qu'il y a de beau dans ces révélations » (p. 292). Flaubert, dans sa correspondance, déclare que « [c]e qui [l]'indigne [le plus] ce sont ceux qui ont le bon Dieu dans leur poche et qui vous expliquent l'incompréhensible par l'absurde » $(1975$, p. 171), ce qui correspond tout à fait à sa représentation du swedenborgisme. Il écrit, par exemple, que Swedenborg, en un an, a « exploré Vénus, Mars, Saturne et vingt-trois fois Jupiter. 
De plus, il a vu à Londres Jésus-Christ, il a vu saint Paul, il a vu saint Jean, il a vu Moïse, et, en 1736, il a même vu le Jugement dernier » (p. 291). En bref, le swedenborgisme, éphémère et introspectif, est soumis à une ironie qui souligne surtout son côté invraisemblable et comique, sans laisser aucune place à une interprétation favorable.

En revanche, le magnétisme, les tables tournantes et même la magie reçoivent un meilleur traitement il leur est consacré un plus grand nombre de pages. Ces trois pratiques ont un trait en commun: l'accessibilité. Le magnétisme, les tables tournantes et la magie sont plus facilement abordables par un lectorat marginalement critique. De plus, le magnétisme s'abrite sous l'étiquette de "science", impliquant des effets visibles qui sont difficiles à expliquer autrement. La présentation qui en est faite contraste favorablement avec celle du swedenborgisme, ce qui peut laisser croire à une forme de sympathie de la part de Flaubert pour ces pratiques.

\section{La contradiction, le conflit et le scepticisme}

Les divers épisodes du roman sont structurés au moyen de trois procédés : une contradiction d'origine intertextuelle, un conflit idéologique et une résolution empreinte de scepticisme. L'ensemble stigmatise une méthode non scientifique et confuse, qui court à travers la structure cyclique de l'œuvre et illustre l'incapacité qu'ont Bouvard et Pécuchet d'apprendre de leurs erreurs.

La contradiction se nourrit surtout de la lecture de livres contradictoires (voir Meyer, 1994, p. 40). L'emploi de ce procédé, très marqué dans certains segments, notamment celui 
sur la taille des arbres, n'est pas exploité dans celui du paranormal. Par exemple, au lieu de remettre en question les tables tournantes en les ridiculisant ou en soulevant des contradictions théoriques, Flaubert affirme par la voix du narrateur : «Le phénomène des tables tournantes n'en est pas moins certain» (p. 280), malgré le fait que les experts s'opposent, chacun avec une théorie différente, pour expliquer le phénomène. La présentation des théories est agencée de manière à montrer que nombre de scientifiques connus s'interrogent sérieusement sur la nature d'un phénomène dont l'existence serait cependant certaine : « Le vulgaire l'attribue à des Esprits, Faraday au prolongement de l'action nerveuse, Chevreul à l'inconscience des efforts, ou peut-être, comme admet Ségouin, se dégage-t-il de l'assemblage des personnes une impulsion, un courant magnétique? » (p. 280). Le narrateur, au lieu de dresser les diverses théories les unes contre les autres, ne favorise aucune des solutions, indiquant plutôt qu'il y a matière à poursuivre les recherches avant de résoudre le mystère des tables tournantes, neutralisant de cette manière la possibilité d'une contradiction qui pourrait mettre en cause le statut du paranormal.

Similairement, sur le plan des personnages, le conflit idéologique entre Bouvard et Pécuchet sur la question du magnétisme, très marqué au début, cède rapidement le pas à un accord. Bouvard, le plus sceptique des deux, se laisse convaincre et devient le magnétiseur préféré, celui dont la «réputation parvint jusqu'à Falaise » (p. 282). Après la conversion de Bouvard, le médecin Vaucorbeil demeure le seul personnage à être sceptique. Vaucorbeil commence par appeler le magnétisme «un tas de jongleries, [...] dont les effets proviennent de l'imagination » (p. 283), mais face à la réussite 
d'une expérience magnétique, il annonce : ce n'est qu'«[u]ne coïncidence, parbleu! Ça ne prouve rien», mais en poursuivant: «Ne continuez plus! ce sont des amusements dangereux!» (p.290). Par cette dernière exclamation, Vaucorbeil confirme, avec toute son autorité de médecin, que le magnétisme existe et qu'il est suffisamment puissant pour présenter un danger public. Quant à la magie, personne ne doute de la capacité de Bouvard et Pécuchet à devenir magiciens, et à la suite du départ terrifié de leur domestique Germaine, suscité par l'observation clandestine de leurs activités occultes, ils « inspir[en]t une vague terreur » (p. 296) à la communauté. À la fin du segment sur le paranormal, aucun des personnages représentés dans le texte ne demeure sceptique.

La dernière manifestation de scepticisme est celle du narrateur, qui se mêle aux voix des personnages en style indirect libre. Par exemple, à la suite de la réussite d'une guérison magnétique, le narrateur commente le succès en disant que «ce n'était pas l'effet de l'imagination, certainement » (p. 284). Le narrateur modalise le doute par la négation, suggérant que celui-ci n'était pas fondé. Jusque-là, le narrateur soutient donc la validité du magnétisme. Mais en qualifiant la négation par «certainement», le narrateur réinsère l'incertitude tout en refusant d'appuyer une théorie plus qu'une autre, technique très répandue à travers le texte.

Le narrateur ne se limite pas à une ambiguïté passive. Dans l'introduction du segment sur les tables tournantes, il écrit ainsi : «La Presse offrant avec sérieux ces bourdes au public, le renforçait dans sa crédulité » (p. 277). Comparons cela avec la déclaration étonnante sur la certitude du 
phénomène des tables tournantes quelques paragraphes plus loin, et la position du narrateur semble contradictoire. L'ironie flaubertienne n'est donc pas absente de ce segment, mais elle est moins apparente, plus latente qu'ailleurs dans le roman. L'ambiguïté de la prise de position du narrateur permet au paranormal de parler pour lui-même avec tout ce qu'il comporte de ridicule. Cela dit, le manque de contradictions intertextuelles, si présentes dans d'autres segments, et le fait que tous les personnages se laissent convaincre des pouvoirs des tables tournantes, du magnétisme et de la magie, semblent indiquer un penchant favorable envers le paranormal.

\section{Réussites et défaites}

Le résultat des expériences en magnétisme est ambigu. La plupart des expériences se terminent de manière non concluante. Cependant, il y a deux cas où l'issue est soit une coïncidence, soit une réussite marquée. Dans le premier cas, Bouvard et Pécuchet entreprennent de guérir une vache souffrant de gonflements. Après quelques passes magnétiques énergétiques, la vache se vide de manière violente et guérit. Il est impossible de savoir si les deux bonshommes sont responsables de la guérison de la vache. La réussite la plus inexplicable se manifeste en présence du médecin Vaucorbeil, radicalement sceptique. Bouvard magnétise une jeune hystérique et le médecin l'interroge sur l'activité présente de sa propre femme. Quelques pages plus loin, quand Bouvard, Pécuchet et tous leurs invités sont sur le point de condamner le magnétisme, il est révélé que la femme du médecin se livrait précisément à l'activité énoncée par l'hystérique («Hein? 
quoi? Ah! j'y suis. Elle coud des rubans à un chapeau de paille.»). Flaubert ne donne aucune suggestion quant à la manière dont l'hystérique aurait pu connaître la réponse à la question du médecin, si ce n'est qu'elle serait une véritable somnambule voyante.

Le succès de l'hystérique magnétisée est d'autant plus étonnant qu'il n'y a pas de réussites semblables ailleurs dans le livre. Quand les deux compères se mettent à faire des conserves, les bocaux explosent dans la cave. Quand ils se livrent à l'agriculture scientifique, une botte de foin pourrit et prend feu. Pécuchet perd sa virginité et contracte une maladie transmise sexuellement. Les aventures de Bouvard et Pécuchet sont marquées surtout par des défaites, parfois catastrophiques, à l'exception d'un incident pendant l'épisode de médecine au troisième chapitre. Mme Bordin, souffrant d'une tache d'herpès au visage, que le médecin Vaucorbeil traite sans succès depuis quelques mois, accepte d'être soignée par Bouvard et Pécuchet. Ceux-ci prescrivent, contre les conseils de Vaucorbeil, un médicament redoutable: «dans les doses permises et malgré l'effroi du mercure ils administrèrent du calomel. Un mois plus tard, Mme Bordin était sauvée. » (p. 129) Ce succès apparent est diminué par le fait que l'herpès ne se manifeste que périodiquement et que les taches peuvent guérir toutes seules. De plus, Bouvard et Pécuchet utilisent un médicament à la base de mercure, un remède démodé et généralement accepté comme étant toxique. La définition de mercure dans Le Dictionnaire des idées reçues - «Tue la maladie et le malade» (1990, p. 256) - nous éclaire sur l'opinion de Flaubert à ce propos. 
Loin de rétablir un équilibre, les échecs subis par Bouvard et Pécuchet dans leurs aventures occultes sont bien moins significatifs que leurs réussites. Les deux protagonistes, essayant d'appeler l'âme du défunt père Bouvard par la magie, sont interrompus avant de pouvoir conclure la cérémonie. L'âme du père Bouvard n'apparaît nullement, ce qui indiquerait une défaite, bien que l'auteur n'exclue pas une réussite de l'expérience, eût-elle été menée à son terme (voir Calvez, 2000, p. 100). Le fait que les réussites sont plus marquantes que les défaites suggèrerait une indulgence surprenante de la part de Flaubert envers le paranormal, ce qui n'est cependant pas confirmé par sa correspondance.

\section{La correspondance}

Même s'il est facile de relever des attitudes négatives envers le paranormal à travers la correspondance de Flaubert pendant qu'il mène ses recherches, sa conception du paranormal est difficile à définir après lecture du huitième chapitre. L'auteur commence par traiter le paranormal de « défilé d'absurdités [...] vraiment attristant» et «inouï d'imbécillité », mais cette aversion ne se limite pas au paranormal. Flaubert inclut le magnétisme à la religion et à la philosophie sous l'étiquette de «tas de bêtises» (Flaubert, 1971, p.171) dans sa correspondance avec Madame des Genettes. Il écrit à Edmond de Goncourt qu'il essaye de rendre la séquence «claire et vivante », ce « qui n'est pas une petite besogne » (1971, p. 231). Après avoir mis ses lectures paranormales de côté et après la rédaction $\mathrm{du}$ huitième chapitre, le ton sceptique de sa correspondance change. Flaubert écrit gaiement qu'il se fait 
traiter de "grosse fille hystérique» (1971, p. 198) et il se dépeint quelques semaines plus tard comme étant pris d'un "goût hystérique » (1971, p. 271) pour des harengs. Il y a bien des moments, selon Meyer, où Flaubert paraît aussi naïf que Bouvard et Pécuchet et tient à croire aussi désespérément au paranormal (p. 41). Les similarités entre Flaubert et les deux protagonistes sont en effet nombreuses : la passion des livres, leur isolation du monde et leur dévotion à leurs travaux écrits - Bouvard et Pécuchet à leur éternel travail de copistes à la fin du roman, et Flaubert à Bouvard et Pécuchet. Cela dit, Flaubert n'est ni Bouvard ni Pécuchet. L'opinion de Flaubert sur l'occulte est très claire; il considérait le sujet comme ridicule et n'hésitait pas à le dire. Pourquoi donc choisit-il de ne pas souligner ce scepticisme dans son roman? Le paradoxe de Milan Kundera rend bien compte de ce phénomène :

Le romancier n'est le porte-parole de personne, et je vais pousser cette affirmation jusqu'à dire qu'il n'est même pas le porte-parole de ses propres idées. Quand Tolstoï a esquissé la première variante d'Anna Karénine, Anna était une femme très antipathique et sa fin tragique n'était que justifiée et méritée. La version définitive du roman est bien différente, mais je ne crois pas qu'entre-temps Tolstoï ait changé ses idées morales. Je dirais plutôt que, pendant l'écriture, il écoutait une autre voix que celle de sa conviction morale personnelle. Il écoutait ce que j'aimerais appeler la sagesse du roman. (p. 190)

Cette théorie pourrait rendre compte du conflit qu'on a relevé entre les croyances de Flaubert et le segment sur le paranormal de Bouvard et Pécuchet.

Le commentaire de Jean-Paul Sartre, voulant que Flaubert " s'empare du monde [littéraire] mais [...] pour le détruire » (voir Flaubert, 1979, p. 35), ne s'applique pas dans le cas de l'occulte. Sur le plan du réalisme et dans la structure du roman, 
le paranormal partage le même traitement que les autres savoirs. Par contre, l'emploi du vocabulaire savant, le traitement négatif du swedenborgisme en comparaison de celui réservé au magnétisme et aux tables tournantes, ainsi que l'accent mis sur les succès des protagonistes plutôt que sur leurs échecs sont autant d'indices d'une relative sympathie de l'auteur vis-à-vis du paranormal. Flaubert écrit à Auguste Sabatier : Bouvard et Pécuchet « est un testament, le résumé de mes expériences et mon jugement sur l'homme et les œuvres de l'homme » (Ton-That, 1999, p. 105). Il est possible, après avoir tellement étudié le sujet et après en avoir travaillé et retravaillé la matière, que le scepticisme flaubertien envers le paranormal se soit adouci non pas parce que l'auteur y croyait, mais parce qu'il pouvait le considérer avec un peu d'indulgence, laissant à ses lecteurs le soin d'établir par eux-mêmes la nature illusoire du sujet. En fin de compte, Flaubert, comme Tolstoï, aurait ici écouté la sagesse du roman, manifestant une sympathie inattendue pour un sujet qu'il considérait comme dérisoire, et lui donnant une complexité et un intérêt supérieurs.

\section{Bibliographie}

CALVEZ, Éric Le. 2000, "Bouvard et Pécuchet magiciens", Nineteenth-Century French Studies, Vol. XXIX, No. 1 et 2, p. 100-136.

ChARLES, Shelly. 2003, «Pigault-Lebrun : tonner contre! Les mauvaises lectures de Flaubert », Littérature, no 131, p. 1836. 
Flaubert, Gustave. 1979, Flaubert et Pécuchet, éd. de Claudine Gothot-Mersch, Paris, Gallimard, coll. « Folio »;

—. 1971, "Correspondance 1877-1880», OEuvres complètes de Gustave Flaubert, t. 16, Paris, Club de l'Honnête homme;

—. 1990, Le dictionnaire des idées reçues, éd. de Marie-Thérèse Jacquet, Paris, Nizet.

KeMPF, Roger. 1990, Bouvard, Flaubert et Pécuchet, Paris, Grasset.

KunderA, Milan. 1986, L'Art du roman, Paris, Gallimard, coll. « Folio ».

MEYER, E. Nicole. 1994, "Facts into Fiction in Flaubert's Bouvard et Pécuchet», Nottingham French Studies, Vol. 33, No. 2, p. 37-46.

Ton-ThAT, Thanh-Vân. 1999, Bouvard et Pécuchet. Une odyssée de la bêtise, Paris, Éditions du temps, coll. « Lectures d'une œuvre ».

WALL, Geoffrey. 2006, « The Invisible Man: An Essay on Flaubert and Celebrity», The Cambridge Quarterly, Vol.35, No.2, p. 133-150.

\section{Résumé}

Plusieurs indices textuels suggèrent que le segment de Bouvard et Pécuchet consacré à l'occulte est écrit avec moins de scepticisme que ceux qui portent sur d'autres savoirs moins controversés. Ce phénomène a de quoi surprendre, étant donné la manière ouvertement critique dont Flaubert se prononce publiquement sur ce sujet. Cette étude analyse ce soupçon de 
sympathie, fort inattendu, pour les tables tournantes et pour le magnétisme et tente de préciser la nature du rapport, paradoxal, qu'entretient Flaubert avec le paranormal.

\begin{abstract}
The section of Flaubert's Bouvard et Pécuchet that focuses on the occult is surprisingly lacking in scepticism compared to many other subjects that merit, perhaps, a more generous representation than they receive. Such favourable treatment is particularly surprising when Flaubert's overt criticism and scepticism of the paranormal is taken into account. This article examines Flaubert's seemingly sympathetic treatment of tableturning and magnetism while attempting to discover the paradoxical relationship between the author and his text.
\end{abstract}

